\title{
Acompañamiento Docente en Proyectos Informáticos de Desarrollo de Software para el Usuario Final en una Institución de Educación Superior
}

\author{
Teaching Support in Computer Software Development Projects for the End User in a \\ Higher Education Institution
}

Araceli Romero-Romero ${ }^{1}$ y Felisa Yaerim López-Botello ${ }^{2}$

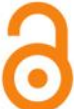 \\ EDICIÓN: 进-CIVTAC}

Recibido: 19/julio/2020

Aceptado: 6/agosto/2020

Publicado: 25/septiembre/2020

特 México

${ }^{2}$ México

\section{IIIIInstitución}

${ }^{1}$ Universidad Pedagógica Nacional ${ }^{2}$ Universidad Autónoma del Estado de México

\section{Correo Eletrónico}

1chelitos_2@hotmail.com 2fely_yaerim@hotmail.com

\section{ORCID}

1https://orcid.org/0000-0002-0328-0525 ${ }^{2}$ https://orcid.org/0000-0003-1732-4979

\section{Citar así: CCAPA / IEEE}

Romero-Romero, A. \& López-Botello, F. (2020). Acompañamiento Docente en Proyectos Informáticos de Desarrollo de Software para el Usuario Final en una Institución de Educación Superior . Revista Tecnológica-Educativa Docentes 2.0, 9(2), 212-222.

https://doi.org/10.37843/rted.v9i2.166

A. Romero-Romero y F. López-Botello, "Acompañamiento Docente en Proyectos Informáticos de Desarrollo de Software para el Usuario Final en una Institución de Educación Superior ", RTED, vol. 9, n. ${ }^{\circ} 2$, pp. 212-222, sep. 2020.

\section{Resumen}

La mayoría de los proyectos informáticos de desarrollo de software no terminan en el tiempo o costo estimado, además el usuario final no queda completamente satisfecho con el producto entregado, por lo anterior el objetivo fue exponer de forma documental cuales son los factores de éxito y fracaso de los diseños, además cómo estos factores relacionan al usuario final y el impacto en su desarrollo. Como fuente de información se analizaron 15 documentos, reportes publicados por The Standish Group de 1994 hasta 2015, por ser una organización encargada de estos estudios, además del reporte "Chaos Report", el cual proporcionó una visión global de estadísticas de procesos informáticos. El método de recopilación de estudios existentes permitió un análisis de causas documentadas del fracaso; posteriormente pudo clasificar causas relacionadas con el usuario o cliente final, sobresaliendo: falta de participación adecuada, escasez de involucramiento, carencia en retroalimentación, usuarios con pobres habilidades de comunicación, expectativas poco realistas del usuario, falta de gestión de expectativa final, requerimientos incompletos, carencia de actitudes y rechazo al cambio. Como aportación final, presentó un procedimiento a través del acompañamiento docente como herramienta para resolver problemas a través de enseñanza práctica en proyectos realizados por estudiantes de una IES para el cliente final, tomando en cuenta las recomendaciones propuestas y los factores que originan el fracaso de los planes, garantizando que el usuario final sea parte del éxito.

Palabras clave: Acompañamiento docente, desarrollo de software, factores de fracaso, usuario final.

\section{Abstract}

Most of the computer software development projects do not finish in the estimated time or cost, in addition the end user is not completely satisfied with the delivered product, therefore the objective was to expose in a documentary way what are the factors of success and failure of the designs, as well as how these factors relate to the end user and the impact on their development. As a source of information, 15 documents were analyzed, reports published by The Standish Group from 1994 to 2015, as an organization in charge of these studies, in addition to the "Chaos Report" report, which provided a global view of statistics on computer processes. The method of compiling existing studies allowed an analysis of documented causes of failure; Later, he was able to classify causes related to the end user or customer, standing out: lack of adequate participation, lack of involvement, lack of feedback, users with poor communication skills, unrealistic user expectations, lack of final expectation management, incomplete requirements, lack of attitudes and rejection of change. As a final contribution, he presented a procedure through teaching accompaniment as a tool to solve problems through practical teaching in projects carried out by students of an IES for the final client, taking into account the proposed recommendations and the factors that originate the failure of the plans, ensuring that the end user is part of the success.

Keywords: Teaching support, software development, failure factors, end user. 


\section{Introducción}

Si bien en los últimos años se ha hecho mucho hincapié en adoptar y adaptar metodologías de administración de proyectos en los desarrollos de software, asegurando el éxito de estos; muchas veces los responsables de la administración centran demasiado su atención en otros aspectos, dejando aparte al usuario; causando problemas en procesos o retrasos al presentar el producto final, generando muchas veces inconformidad en el consumidor al no satisfacer sus necesidades. Por lo tanto, es importante identificar como se relaciona el usuario o cliente con el éxito o fracaso del producto final; en consecuencia, los diseños informáticos de desarrollo con software tienen dependencia al generar un método incluyente con el cliente final en los procesos informáticos de desarrollo de software de una forma correcta asegurando el éxito de estos.

De igual forma, haber examinado cuales factores relacionan usuarios finales, en correlación con el impacto sobre el éxito o fracaso de los procesos, respecto al resultado del análisis obtenido, se propuso un método direccionado a integrar al usuario final o cliente en los diseños informáticos generadores hacia el éxito. Algunos beneficios por alcanzar en futuros administradores o líderes de proyectos al tomar decisiones es integrar al usuario final al momento de iniciarlo o desarrollarlo; con acompañamiento docente, siendo herramienta para resolver problemas mediante enseñanza práctica, realizados por estudiantes de una IES; tomando en cuenta recomendaciones propuestas relacionadas por los factores causantes del fracaso, garantizando así que el usuario final sea parte del éxito.

Los objetivos fueron definir un método, permitiendo integrar al cliente final en proyectos de desarrollo con software, a través del acompañamiento docente como herramienta para resolver problemas mediante enseñanza práctica en proyectos realizados por estudiantes en una IES; así mismo examinar factores de éxito y fracaso, determinando la manera cómo se relacionan con el usuario final.

\section{Desarrollo}

Hoy en día es imposible concebir una empresa exitosa sin apoyo de Tecnologías de Información y la Comunicación (TIC), hacia administrar sus procesos de negocio (Saavedra \& Tapia, 2013). Tecnologías de la Información (TI) son adoptadas no solo por empresas sino también por el entorno social, su influencia crece notablemente en aspectos económicos nacionales, así como en competitividad del país; ha demostrado una analogía positiva, además contundente entre ambas variables; en países desarrollados como en países en vías de desarrollo (Dirección General de Innovación, Servicios y Comercio Interior [DGISCI], 2015). Respecto al impacto de TIC en el bienestar económico, también social sólo existen cuando éstas se incorporaron a actividades cotidianas de personas, además de agentes económicos de un país.

Un país competitivo se caracteriza por tener altos grados en desarrollo sostenido respecto al Producto Interno Bruto (PIB) per cápita. El Instituto Mexicano para la Competitividad (IMCO) el tema de competitividad es capacidad en los países dirigido a atraer, así como retener inversión, al mismo tiempo talento (Palacios, Flores-Roux \& García, 2013). Por parte del PMI, administración de diseños es logrado mediante una adecuada aplicación e integración de cuarenta y siete procesos en gestión de proyectos, agrupados lógicamente por cinco grupos. Estos cinco grupos son: Iniciar, Planificación, Ejecución, Seguimiento, Control y Cierre (PMI, 2013).

En Chamoun (2002) y el PMI (2013) definen cinco procesos en administración de proyectos:

1. Inicio. Establece visión, misión por cumplir, objetivos, restricciones al mismo tiempo supuestos.

2. Planeación. Desarrolla un plan que ayude a lograr objetivos. establecen estrategias, con énfasis en prevención, evitando improvisación.

3. Ejecución. Llevar a cabo acciones requeridas, implementar, contratar, administrar e integrar un equipo con lo planeado.

4. Control. Comparar lo ejecutado o lo real contra lo planeado (control), de no contar con desviaciones se continúa con ejecución, de lo contrario se toman acciones correctivas, para continuar con el proceso de ejecución.

5. Cierre. Concluir, además de cerrar relaciones contractuales, se elaboran los documentos con los resultados finales, archivos, evaluaciones, así como lecciones aprendidas, entre otros. 
Los proyectos informáticos según Guerra \& Bedini (2005) bajo definición establecida por el PMI, y Chamoun entre otros, se caracterizan por el impacto al sufrir obsolescencia, especialmente tecnológica e intensa participación con recurso humano de distintas áreas en la organización durante su desarrollo. El software es el corazón de cualquier operación en una organización, prácticamente está relacionado con su operación, aunque en muchas organizaciones lo que respecta a actividades informáticas son consideradas una actividad de apoyo.

El impacto referente a TI es muy alto. Algunas organizaciones consideran a sus diseños de TI como estratégicos, por lo que están alterando su estructura, operación e intentando adaptarse al mercado, pero también aprovechando ventajas en su coordinación, registro y análisis de información; el apoyo para toma de decisiones brindando por los sistemas de información logra una ventaja competitiva. En cuanto a industria del software, ésta es una industria emergente en proceso de consolidación. Sus procesos en producción se encuentran inmersos en un ambiente con rápido cambio, similar a lo que respecta a organizaciones con mucha presión. Desgraciadamente, respecto a calidad del software no tiene una trayectoria tan brillante como su crecimiento (Zavala Ruiz, J. \& Jesús María, 2004), (Ruiz, 2004):

\begin{abstract}
Organizaciones consideran los proyectos de software como cualquier otro proyecto, a pesar de tener un mayor impacto en los aspectos estructurales en su operación. También consideran deben ser sencillos, también baratos. Es frecuente cuando la empresa consultora se vea obligada a asumir el costo del diagnóstico, considerando respecto a etapa de desarrollo "recuperará lo perdido". Sin embargo, dependiendo del proceso y su complejidad, puede salirse de control e incluso tornarse en un fracaso por falta de un diagnóstico organizacional adecuado (p. 9).
\end{abstract}

Uno de los grandes problemas del entorno industrial del software, a pesar de contar con estándares, metodologías, técnicas, lineamientos, incluso con herramientas, éstas no se emplean de manera generalizada, haciendo de esta industria una artesanía. Además, los profesionales en software en su gran mayoría tienen deficiencias académicas importantes, además muchos son generalistas (o todólogos) en vez de especialistas. Bajo los esquemas ad hoc, la industria no puede repetir ni predecir el proceso de producción, ni estimar en cuanto a calidad del producto final (Ruiz, 2004).

Los usuarios en procesos informáticos son personas, pero no procedimientos ni técnicas, referidas como críticas logrando el objetivo, lo anterior retomado por Gido \& Clements (1999). Los procedimientos y técnicas son herramientas, ayudan a personas a realizar sus trabajos. Involucrados a cumplir los procedimientos está el gerente, el cual coordina actividades del equipo. Otro involucrado clave es el equipo de proyectos, al ser un grupo asignado a trabajar en cooperación bajo un objetivo. El ayudar a estas personas a desarrollarse, así como crecer para convertirse en un equipo unido también efectivo requiere esfuerzos por parte del gerente, además de cada miembro del equipo.

En Rodríguez (2015) se plantea un porcentaje considerable de fracasos informáticos debido al hecho de no contar con un usuario-final calificado. En su trabajo se hace un estudio del impacto de aptitudes del usuario-final en el éxito o fracaso de los desarrollos de Sistemas informáticos (SI). Respecto a principales aptitudes son conocimientos, competencias profesionales, así como organizacionales.

El éxito y el fracaso en los procesos informáticos analizados por varios autores, como Pereira, Cerpa \& Rivas M. (2004) han expuesto como una cuestión de percepción, tendiente a variar. En un estudio realizado a varios diseños informáticos, los únicos criterios del éxito unánimes entre las partes involucradas fueron "cumplir con requerimientos del usuario, conseguir el propósito, cumplir con tiempo de entrega, cumplir el presupuesto, dejar satisfechos a los usuarios y obtener un software de calidad" (p.79).

Hidding \& Nicholas (2009) distinguen dos escuelas del pensamiento. Estas escuelas son respecto a eficiencia, así como eficacia. Escuela del pensamiento de eficiencia, define el éxito en términos hacia una administración de los proyectos tomando como criterios el costo, tiempo, etc. mientras la escuela del pensamiento de eficacia, define el éxito en términos con los resultados del proyecto, por ejemplo: ganancias, además beneficios, es decir, toma una vista externa, se centra en los resultados, pero también en partes interesadas.

Por lo anterior, un diseño completado debajo del presupuesto en menos tiempo establecido no es necesariamente exitoso, de igual forma completado 
por encima del presupuesto sobre el tiempo no necesariamente es un fracaso, sino depende del enfoque. Humphrey (2005) considera exitoso, aquel completado entre el $10 \%$ más o menos de lo acordado en costo, en tiempo, además se proporcionó bajo funcionalidades acordadas; de lo contrario cuando no se entregó nada es considerado un fracaso. The Standish Group dice, es exitoso si es completado en tiempo, presupuesto, además con todas sus características, así como funciones specificadas (The Standish Group, 1994).

Humphrey (2005), también otros autores como Standish Group mencionan otra clasificación intermedia entre un exitoso y fracasado, a los llamados desafiantes (challenged projects) esta clasificación establece que fueron terminados severamente tarde o por encima del presupuesto establecido o tienen funciones muy reducidas. Para Standish Group, este tipo fueron completados, funcionaron, pero sobrepasaron el presupuesto, además el tiempo estimado; ofrecieron solo algunas características, también pocas funciones originalmente especificadas.

Para Charette (2006) los fracasos forman parte de la competitividad en los negocios y es esperado su acontecimiento. Si no se falla lo suficiente ahora, entonces probablemente no está haciendo nada por competir. Explica respecto a fallar correctamente, lo cual significa una organización tendiente a tomar riesgos adecuados, consciente de esos riesgos, pero también consciente a mitigarlos. Cuando se aprende de los fracasos, pueden convertirse en el progreso.

Al seguir correctamente una administración de procesos, probablemente resulte exitoso al final, Horine (2013) menciona desde un punto de vista académico, además utópico la definición de un proyecto exitoso, como los entregables prometidos, completados en tiempo dentro del presupuesto, donde todos los entregables cumplen con especificaciones de rendimiento, calidad, cumpliendo con propósito, objetivos originales, expectativas de los interesados y relaciones de ganar-ganar.

Respecto a los fracasos en procesos informáticos según Markus (como se citó en Ruiz, 2004) el fracaso de software es bastante limitado, considera los fracasos de manera genérica como "fallas" (p. 43). También considera a los fracasos, factores fuera de control del administrador del proyecto, así como del equipo (p.46). Se identifica como causas origen "carencia de compromiso del personal de ventas, mal entendimiento de quienes eran los clientes reales, conflicto entre necesidades del cliente desconocidas por equipo de diseño, falta de un staff calificado, alta rotación del staff y lo referente a percepción negativa que los usuarios tienen de su departamento de informática" (Ruiz, 2004, p. 90).

Pereira, Cerpa \& Rivas (2004), los fracasos en proceso de software son problemas con la estimación, programación de actividades y coherencia entre requerimientos, También fallas en: comunicación con el cliente/usuario, estructura organizacional, liderazgo, apoyo del nivel gerencial, esfuerzo, choques de personalidades; uso inefectivo respecto a métodos en desarrollo de software, procesos de negocios, recursos inapropiados, gestión de diseños y herramientas de seguimiento inadecuados.

El término "fracaso", según Charette (2006) debe reservarse a proyectos con una buena oportunidad de éxito administrados profesionalmente, por lo anterior, todo lo demás debe ser etiquetado como equivocaciones. En otras palabras, una organización, además de las partes interesadas saben en qué están trabajando, hacen todo para aumentar sus posibilidades de éxito dadas las limitaciones. Las tasas de fracasos en los procesos de IT dependen del tamaño, mientras más grande sea, mayor probabilidad al fracaso (Hidding $\&$ Nicholas, 2009). Humphrey (2005) menciona un proyecto de software a gran escala es inherentemente inmanejable, además tiene más probabilidad de fracasar.

El problema de visibilidad de un administrador de diseño en cierto momento no se puede decir dónde está, debido a que la información muchas veces no es visible, esto es, mientras los desarrolladores pueden superar pequeños problemas presentados, estos problemas agregan cargas de trabajo, así como retardos, pero con el tiempo los problemas aumentan poco a poco y tarde o temprano el proyecto enfrenta serios problemas no observados con anticipación. En procesos muy grandes de software, los administradores no ven los retrasos hasta ser muy grandes, también obvios, siendo demasiado tarde para hacer algo al respecto (Humphrey, 2005).

Otro termino importante es cancelados, Boehm (2001) lo considera un diseño fracasado, falso, también peligroso. Hay varias acepciones de cancelado las cuales son, cuando una gran 
cantidad de programas de software son iniciados apropiadamente, bien administrados, terminados antes de su finalización, pero sus supuestos originales han cambiado. Cuando sus administradores tienen la impresión de un desperdicio de recursos para la empresa es otra acepción de cancelado y peligroso. Sin embargo, a los administradores, la cancelación los convierte en directores de un fracaso, por lo cual prefieren quedarse callados o no decir nada, siguiendo adelante y en busca de otro proyecto.

Otros factores relacionados a cancelación son falta de soporte ejecutivo, así como cambios de requerimientos. Un proceso bien administrado en un ambiente de constantes cambios debería ser terminado cuando los continuos costos de adaptación son mayores a los beneficios. Otros serian falta de planeación, necesidades, administración de IT y analfabetismo tecnológico (Boehm, 2001). Por su parte Charette (2006) establece que la mayor parte de cancelaciones no son verdaderos fracasos, por lo contrario, representan equivocaciones.

Boehm (2001) propone cinco pasos a fin de terminar un proyecto sano y rentablemente, el primero hace referencia a vender el producto; es decir, hacer cuestionarios de satisfacción, necesidades del usuario, asegurar el involucramiento del usuario en definición del producto, hacer ejercicios de prototipos, por último, su revisión. El segundo concierne a usar una administración del riesgo e incrementar el compromiso, integrar el modelo de administración del riesgo, el cual provee un efectivo marco de trabajo evitando el riesgo y controlando. El tercero es hacer tableros de revisión de arquitectura y razones de viabilidad, este se centra en el mejor talento de organización técnico, administrativo, también del usuario para una revisión exhaustiva de su arquitectura, así como viabilidad del negocio en dos puntos de compromiso de los interesados críticos.

El cuarto paso es supervisar premisas del negocio, frecuentemente unas organizaciones establecen proyectos basados en premisas sujetas a ser o no verdaderas con el tiempo, cuando una premisa llega a ser invalida es esencial juntar a los interesados para una revisión respecto a continuar o terminar lo propuesto. El quinto paso corresponde a no comparar la terminación con el fracaso, en este paso es necesario identificar, como terminar procesos poco factibles tempranamente.

El éxito de lo anterior puede desarrollarse a través del trabajo dentro de la escuela, acompañamiento docente como herramienta para resolver problemas por medio de una enseñanza práctica, sin limitarse a impartir o compartir información, sino buscar desarrollar en los estudiantes destrezas que les permita resolver problemas bajo necesidades actuales. Una educación no consiste solamente en transmitir conocimientos, sino en proporcionar al estudiante un conjunto de situaciones de aprendizaje, contribuyendo al crecimiento integral, estimulando el desarrollo de procesos cognoscitivos, facilitando una comprensión, además favoreciendo una transformación del mundo.

De acuerdo con Mujica, (2014) la acción del docente es fundamental en educación, no hay organización didáctica capaz de sustituirlo. A pesar de todas las nuevas concepciones pedagógicas, el docente sigue siendo indispensable, además pieza fundamental en el proceso educativo. Un buen docente logra en sus educandos crecimiento, cuando éstos quieren aprender, compartir inquietudes y generar nuevas ideas. Favoreciendo este crecimiento a través del enfoque crítico reflexivo y del acompañamiento docente; este enfoque se origina en un marco humanista, así como social en el que convergen numerosos aportes teóricos como las investigaciones sobre el pensamiento del profesor; etnografía educativa (Rockwell, 1995; Achilli, 2001); teoría crítica en educación (Jackson, 1998; 2002; Angulo Rasco, 1999); teorías del aprendizaje adulto (Mezirow, 1981; Tennant, 1991); investigaciones sobre el aprendizaje situado, en contexto (Chaiklin \& Lave, 2001), pedagogía crítica de Paulo Freire, entre otros.

El asesoramiento proporciona sugerencias durante el acompañamiento, mejora el desempeño docente y se sustenta en experiencias; también a través de los conocimientos adquiridos por el acompañante durante su propia práctica pedagógica por medio de reflexión práctica reformulada, además de su validación, el sentido de observación, así como del análisis colectivo de los docentes, logra fortalecer una pertenencia a un grupo de aprendizaje, una comunidad tendiente a ofrecer, generar oportunidades y medios de enseñanza aprendizaje.

\section{Metodología}

El análisis de los documentos abordó el éxito y fracaso de los proyectos informáticos; respecto al muestreo fue no probabilístico de acuerdo con la 
selección, así como de su disponibilidad. Por lo anterior, no fue necesario aplicar instrumentos directamente, pero los documentos observados y analizados hicieron uso de instrumentos, permitiendo contemplar dimensiones: perfiles de procesos, seguimiento, encuestas individuales, encuestas grupales, entrevistas de casos, estudios generales, además autopsias de proyectos. Una vez determinadas las causas documentadas de los fracasos informáticos, analizado su contenido, usando escala de medición nominal, además ordinal (mediciones cualitativas) e identificadas algunas causas, se eligieron aquellas relacionadas con el usuario final; asignándoles un orden basado en su importancia, además con su frecuencia presentada en diversos estudios.

\section{Resultados}

\section{Factores de Éxito y Fracaso}

Como primera fuente de información respecto a documentos, fueron reportes publicados por The Standish Group, por ser una organización líder en hacer estudios de este tipo de investigaciones, además reporte "Chaos Report" proporcionó una visión global estadística de proyectos, con tendencia a tener una mayor concentración en Estados Unidos, así como Europa. El objetivo de Chaos Report es documentar fracasos en el desarrollo de iniciativas TI y encontrar sus principales causas, para evitarlos. Estos reportes abarcan un número diverso de industrias además de organizaciones.

Esta organización clasificó procesos en tres tipos de resolución, primero los exitosos, son aquellos realizados en tiempo como presupuesto, tomando en cuenta características, además funciones especificadas. Los segundos son desafiantes o problemáticos (challenged) terminados fuera de presupuesto o tiempo, además ofrecen menos características, así como funciones. El ultimo son deficientes, debido a no entregar nada, y cancelar en algún punto (The Standish Group, 1994).

A continuación, en la tabla 1, están los porcentajes relacionados a resolución de proyectos a través de años conforme al análisis Standish group y también una gráfica con porcentajes de resolución realizada bajo análisis Standish group, posterior a ellas hay una descripción.

\section{Tabla 1}

Porcentaje de resolución de proyectos a través de los años de acuerdo con Standish group.

\begin{tabular}{|c|c|c|c|c|c|c|c|c|}
\hline$(\%)$ & 1995 & 1996 & 1998 & 2000 & 2002 & 2004 & 2006 & 2008 \\
\hline Exitosos & 16.2 & 27 & 26 & 28 & 34 & 29 & 35 & 32 \\
\hline Problemáticos & 52.7 & 33 & 46 & 49 & 51 & 53 & 46 & 44 \\
\hline \multirow[t]{2}{*}{ Deficientes } & 31.1 & 40 & 28 & 23 & 15 & 18 & 19 & 24 \\
\hline & 2009 & 2010 & 2011 & 2012 & 2013 & 2014 & 2015 & \\
\hline Exitosos & 28 & 37 & 29 & 27 & 31 & 28 & 29 & \\
\hline Problemáticos & 49 & 42 & 49 & 56 & 50 & 55 & 52 & \\
\hline Deficientes & 23 & 21 & 22 & 17 & 19 & 17 & 19 & \\
\hline
\end{tabular}

Nota. Porcentajes relacionados a resolución de proyectos, elaboración propia (2019).

Los porcentajes de resolución a través de los años son asociados a cada clasificación desde 1995 hasta el 2015, por ser la última información a la que tuvo acceso el investigador. Se observó en tabla 1, un mayor porcentaje en resoluciones problemáticas del $56 \%, 15 \%$ de porcentaje menor en resoluciones deficientes, seguido a los resultados de resolución exitosa con un porcentaje $6.2 \%$.

\section{Figura 1}

Gráfica del porcentaje de resolución de proyectos a través de los años de acuerdo con Standish group.

\section{Resolucion de proyectos}

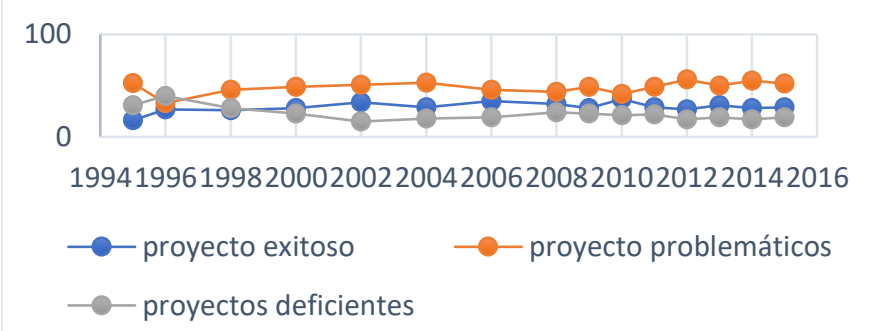

Nota. Resolución de proyectos, elaboración propia (2019).

Respecto a lo anterior, existe inclinación mayor a proyectos con una resolución problemáticos y solo un pequeño porcentaje exitosos, a continuación, hay un análisis causal de un proceso exitoso a fin de tomarlo en cuenta. También analizó el investigador a detalle solo tres reportes de Standish Group, por tener acceso a ellos de manera gratuita, reflejando el comportamiento a lo largo del tiempo. Por lo general en cada reporte existe 10 factores de éxito, así como de fracaso.

Respecto a los factores de éxito únicamente se analizó tres principales factores de cada reporte por ser los más importantes, buscando encontrar cuales de estos factores tenía relación al usuario, los demás 
puntos no tenían correlación directa con el usuario final. El primer reporte analizado fue el del 2009, encontrándose tres principales factores de éxito, los cuales fueron: participación del usuario; Apoyo Ejecutivo; concluyendo con Objetivos claros de Negocio.

El siguiente reporte analizado fue Chaos Manifesto 2013, centrándose en recopilación informativa sobre casos de proyectos reales en ambientes respecto a TI y software. Utilizándose ocho instrumentos diferentes, los cuales incluyeron: perfiles, seguimiento, encuestas individuales, encuestas grupales, entrevistas de casos, estudios generales, autopsias de estos, entre otros instrumentos. Los tres primeros factores de éxito representaron el 50\%, estos factores fueron: Apoyo de administración ejecutiva; Participación de los usuarios; por último, Optimización.

El reporte del Chaos Manifiesto 2013, mostró claramente los resultados carentes de participación de los usuarios, funcionando mal. Una buena participación del usuario tiene un efecto importante en resolución de diseños grandes o pequeños. Otro factor de éxito fue lo relativo a optimización. Definiéndose optimización como un proceso con pequeña mano de obra, además de entrega rápida, pudiendo ser el número uno. Respecto al tamaño, así como a complejidad, triunfa sobre todos los demás factores.

El último reporte analizado del The Standish Group fue el del 2015, estudiándose 50000 proyectos alrededor del mundo, siendo los tres primeros factores de éxito: Apoyo de la dirección; Madurez emocional, también participación del usuario. Respecto al apoyo ejecutivo, debe incluirse tanto aspectos financieros como emocionales. Este apoyo fue clave para estimular al equipo, así como lograr el éxito de los procesos.

Por lo tanto, en cualquier grupo, equipo u organización los resultados dependen de habilidades individuales, pero también de los vínculos del equipo. Por último, en lo que respecta a participación de los usuarios, trata de lograrse su implicación tanto en toma de decisión como en procesos de toma de información, incluyendo incorporación de retroalimentación, revisión de requisitos, prototipado, etc. Hastie, Shane \& Wojewoda (2015) y Evergreen PM (2015).

Los puntos importantes del reporte 2015 por el Standish Group son, redefinió el concepto de "éxito" en los proyectos informáticos, anteriormente un diseño exitoso era aquel terminado en tiempo, presupuesto, además de alcance, sin embargo, en alcance no era una buena medida, porque carecía de cualquier medida de los resultados del cliente, muchas veces se cumplían las tres restricciones, pero dejaban al cliente insatisfecho, por lo tanto, reemplazaron el alcance con una medida de valor de percepción del cliente.

Respecto a probabilidad de éxito, parece inversamente relacionada al tamaño, cuanto más grande es el diseño más probable a no ser exitoso. Respecto al uso de una ágil mejora da mayor probabilidad de éxito, independientemente del tamaño, aplicar una ágil mejora sube el porcentaje de éxito del $11 \%$ al $39 \%$ y bajo el porcentaje de fracasos del 29\% al 9\%, Lázaro (2015) y Weber (2015).

Para complementar el estudio, se analizaron diversos trabajos relacionados con el éxito además del fracaso de procesos informáticos, uno de ellos fue el trabajo de Calderón \& Rodríguez (2010) dividiéndose los factores críticos de éxito en dos grupos: primero los relacionados con organización, como los referidos al usuario final. Entre los factores organizacionales analizados están el apoyo directivo, gerencial, asignación de recursos, cultura organizacional, organización informal, gerencia, manejo del cambio, por último TI. Los segundos factores son los críticos relacionados al usuario, sobresaliendo el impacto de actitudes del usuario final, compromiso, participación, responsabilidad, proactividad como un posible rechazo por razones del cambio, entre otros. Igualmente, la satisfacción mostrada por el usuario-final una vez implantado el SI desarrollado.

En conclusión, el consenso entre los administradores de proceso participantes indicó al momento de entregar un sistema, cumplió con los requisitos del cliente/usuario y funcionaron según lo previsto mediante realización de trabajos, proporcionando un sentido intrínseco de calidad, además de logros personales, siendo lo anterior aspectos importantes para considerar como un éxito (Procaccino \& Verner, 2007). Después de analizar los reportes de Standish Group, así como los demás trabajos relacionados se observó las causas o riesgos de los fracasos de proyectos informáticos, clasificándose en dos grupos: los relacionados con los usuarios o clientes y los relacionados con dirección. 


\section{Diagnóstico de Factores Relacionados al Usuario}

Como se mencionó, el objetivo de este trabajo es encontrar una relación existente entre el usuario final y el éxito de los proyectos informáticos, también ver qué problemas se enfrentan al momento de realizar un proceso informático de desarrollo de software, por lo cual se analizó el estudio de Standish Group visualizado en la siguiente tabla 2.

\section{Tabla 2}

Factores de éxito de proyectos informáticos, con base al estudio de Standish Group.

\begin{tabular}{lllll}
\hline 2009 & 2013 & 2015 & & \\
\hline $\begin{array}{l}\text { Participación } \\
\text { Usuario }\end{array}$ & $\begin{array}{l}\text { Apoyo de la } \\
\text { administración } \\
\text { ejecutiva }\end{array}$ & $\begin{array}{l}\text { Apoyo de } \\
\text { dirección }\end{array}$ & & \\
\hline Apoyo ejecutivo & $\begin{array}{l}\text { Participación } \\
\text { del usuario }\end{array}$ & Madurez emocional \\
\hline $\begin{array}{l}\text { Objetivos claros } \\
\text { de negocio }\end{array}$ & Optimización & $\begin{array}{l}\text { Participación } \\
\text { usuario }\end{array}$ & del \\
\hline
\end{tabular}

Nota. Análisis de estudio, elaboración propia (2019).

En los informes de Standish Group analizados en el periodo 2009 a 2015, reflejan tres causas principales del éxito informático relacionadas con el usuario final, las cuales son participación, apoyo de la administración ejecutiva y apoyo directivo; ubicando al usuario como un aspecto importante en el éxito de proyectos informáticos. Así mismo los procesos carentes de participación de los usuarios funcionan mal.

En los informes de Chaos Report, mencionaron aspectos relacionados con participación del usuario, tomando en cuenta habilidades de comunicación, por lo que los procesos problemáticos, así como deficientes incluyeron típicamente a usuarios con pobres habilidades comunicativas. En lo referente a la implicación de los usuarios, se debe lograr tanto en lo respectivo a toma de decisión como en los procesos de toma de información, que incluye incorporación de retroalimentación, revisión de requisitos, prototipo, etc. (Hastie \& Wojewoda, 2015) y (The Standish Group, 2009). Los otros trabajos analizados por Rodríguez (2015), Arnuphaptrairong (2011), Procaccino, Verner y Overmyer (2002); Procaccino y Verner (2007).

Después de analizar, clasificar, además de ponderar los estudios e identificar cuáles son los factores relacionados con el usuario, los más mencionados por los reportes son los siguientes:
1. Usuarios con pobres habilidades de comunicación.

2. Insuficiencia en participación adecuada de los usuarios

3. Falta de involucramiento en cualquiera de las fases del ciclo de desarrollo de software

4. Carencia en actitudes del usuario como compromiso, participación responsabilidad, así como proactividad.

5. Falta de retroalimentación a los usuarios (en revisión de requisitos y prototipado).

6. Escasez de gestión de expectativa final del usuario también clientes para expectativas realistas y rechazo al cambio por parte de los usuarios

\section{Conclusión}

De acuerdo con lo investigado y a las nuevas tendencias, hay mayor probabilidad de obtener un proyecto informático de software exitoso al dividirse en pequeñas partes, existir acompañamiento en todo el proceso por parte del docente, involucrar al usuario en toda fase, existir una metodología ágil, establecer un canal adecuado de comunicación docente estudiante - usuario final, así como establecer claramente los objetivos.

Relaciones entre docente - educando, es de suma importancia porque el maestro se constituye como guía en el acompañamiento, coordina actividades del aprendizaje, propicia en los educandos adquirir conocimientos necesarios, además proporciona herramientas para resolver problemas mediante enseñanza práctica en proyectos realizados por los propios estudiantes. Lo propuesto permitirá integrar al usuario final en los procesos de desarrollo de software, a través del acompañamiento docente como herramienta, resolviendo problemas y analizando casos reales mediante enseñanza práctica, también el docente será facilitador y guía en diseños realizados por estudiantes de una IES. Los principales problemas tratados de resolver con el método propuesto son:

1. Falta de participación adecuada.

2. Escasez de involucramiento en cualquiera fase del ciclo de desarrollo de software.

3. Carencia en retroalimentación de los usuarios (en revisión de requisitos y prototipado)

4. Usuarios con pobres habilidades de 
5. de comunicación.

6. Expectativas poco realistas del usuario.

7. Falta de gestión de expectativa final del usuario, así como clientes.

8. Requerimientos incompletos.

9. Carencia de actitudes del usuario como compromiso, participación responsabilidad, además de proactividad.

10. Rechazo al cambio por parte de los usuarios.

Una administración de proyectos está compuesta por cinco procesos principales: Iniciación, Planificación, Ejecución, Seguimiento, Control, así como Cierre. Estos procesos son necesarios en todo proceso de desarrollo de software. Las metodologías existentes demandan ser secuenciales y sus fases por lo general son: Requisitos, Análisis, Diseño, Implementación, además Pruebas; orientándose en el ciclo de vida de los diseños de software, pudiendo ser en cascada, incrementales y evolutivos. Por lo tanto, el presente trabajo toma en cuenta las anteriores fases, una administración que permite definir de forma correcta como integrar al usuario en todo el diseño.

\section{Proceso de Iniciación - Planificación.}

Es esta fase los involucrados proponen acompañamiento docente, del administrador $\mathrm{y}$ patrocinador, aquí no necesariamente debe involucrarse al usuario final aún, por ser esta fase donde apenas se propone el proyecto y evalúa de acuerdo con su viabilidad. Si es aprobado por la empresa existe autorización, además de recursos para llevarlo a cabo, dando origen al acta. Al terminar este proceso existirá apoyo por parte de la alta dirección por estar aprobado y cumplir los objetivos de una empresa o negocio.

\section{Propuesta para Involucrar más al Usuario.}

Los mejores usuarios para participar en un pequeño proceso son aquellos bien informados acerca de sus áreas, en cuanto menor sea el área más expertos serán los usuarios. Una vez identificados los diversos usuarios, debe agendarse reuniones, a fin de obtener los requerimientos, teniendo una visión clara de lo esperado, y de esta forma involucrar de manera inicial a los diversos usuarios. No debe entrevistarse o agendarse reuniones con todos los empleados, por ser demasiado tiempo dedicado a ello, pero si a personas claves y expertos en su área; por eso se hace hincapié primero en identificar los roles de los usuarios permitiendo seleccionar expertos en su área, obteniendo información muy valiosa.

\section{Propuesta para Requerimientos Incompletos}

Para solucionar estos problemas una vez identificados los usuarios claves o que utilizaran el sistema, es conveniente involucrarlos desde el principio empezando a evangelizar a los usuarios. The Standish Group (2013) menciona en esencia el evangelismo como una forma de marketing, un estilo muy intenso de comunicación. También muchas veces sugiere un cambio organizacional logrando involucrar cambios en actividades, flujos de trabajo y estructuras organizacionales, responsabilidades, colaboración con otros, nuevas habilidades, etc.

Otro problema recurrente con los usuarios finales refiere a la comunicación, por lo tanto, una vez comenzando el proyecto es importante tener una plataforma de comunicación a lo largo del proceso. Los diseños pequeños son apropiados para tener una comunicación más efectiva. En los proyectos grandes existen tres principales causas de retardos, además de desastres, estas son: estimaciones inexactas, informes de estado inexactos, así como datos históricos inexactos (Charette, 2006). Por lo tanto, si es muy grande, lo más conveniente es dividirlo facilitando una comunicación más efectiva.

\section{Proceso de Ejecución}

El proceso de ejecución, coordinado por el administrador, consiste en obtener los productos entregables del proceso, teniendo en cuenta: personas, recursos, además de seguir el alcance definido. De igual manera, este proceso tiene la finalidad de comunicar a los interesados con previa revisión del docente, el estado de los productos y acerca del trabajo realizado. Aquí los involucrados son: administrador del proyecto, patrocinador, cliente o usuario y equipo de trabajo (Rivera \& Hernández, 2010).

La propuesta señalada a continuación da respuesta a la falta de involucramiento, así como de retroalimentación por parte del usuario, siendo: una vez identificados los usuarios claves y finales que usaran el sistema, conviene definir entregas parciales exhibiéndolas al usuario, además de agendar 
reuniones a fin de escuchar retroalimentaciones por parte del usuario, para ver si el diseño fue lo esperado, logrando validar los procesos de negocio. Al involucrar al usuario en este proceso puede asegurarse el desarrollo del nuevo sistema de software, cumpliendo expectativas del usuario, además de cumplir con los requerimientos. Por lo tanto, en la siguiente fase de pruebas la intervención del usuario es necesaria al probar el sistema final validando, sin encontrar problemas de diseño o en su caso lo no esperado.

\section{Proceso de Control - Seguimiento}

Durante este proceso se realiza el control de cambios, así como el análisis, aprobando o rechazando solicitudes de modificaciones al plan del proyecto en todos sus aspectos. Este seguimiento y control incluye estar al pendiente del alcance, cronograma, presupuesto, recurso humano, calidad, además de riesgos. Adicionalmente se administra o cultiva una relación con los interesados, manejando el proceso de comunicación, logrando informar a todos los cambios aprobados, para ser resueltos lo antes posible.

En este proceso el usuario realiza sus pruebas, en caso de encontrar algo no esperado en el documento de requerimientos debe de comunicarlo, a fin de realizar acciones correctivas necesarias. Por lo tanto, aquí debe de haber una buena comunicación con el usuario final por ser el encargado de probar el sistema y pieza importante al tomar acciones; si hay cambios detectados no contemplados, entonces será buen momento para seguir el procedimiento de administración de cambios e informar al usuario de las implicaciones del cambio. Por lo anterior, conviene seguir el plan de comunicaciones establecidas, logrando informar lo detectado y recibir adecuada retroalimentación del usuario, con el fin de evitar problemas de comunicación.

\section{Proceso de Cierre}

El proceso de cierre es realizado por el administrador en estrecha interacción con el cliente, permitiendo terminar todos los procesos asociados con lo solicitado por la administración del proyecto, retomando verificación del alcance en su parte terminal, para realizar la entrega de los productos del proceso y constatar su aceptación formal por el cliente.

Debe entregarse al docente que estuvo durante el acompañamiento una evidencia de entera satisfacción por parte del cliente de los productos o servicios finales recibidos. En esta fase al involucrar a los anteriores actores en todos los procesos anteriores, resulta una retroalimentación adecuada respaldada en un portafolio de evidencias del estudiante $\mathrm{y}$ el producto habrá cumplido sus expectativas, por lo tanto, el cliente dará su visto bueno del sistema sin ningún problema pudiendo decir que el proyecto es exitoso, entregado en tiempo, además del presupuesto estimado.

Como sugerencias para futuros trabajos, relacionados al tema objeto de estudio, se recomienda abordar el éxito de diseños informáticos basados en aspectos de alta dirección como otro factor presentado en el éxito o fracaso, bajo el análisis de los estudios, reportes de Standish Group, además de trabajos relacionados con el tema. También es importante no dejar de lado el acompañamiento docente, siendo herramienta para resolver problemas reales, encaminados a ser resueltos por especialistas en el área, mediante la enseñanza práctica en diseños realizados por estudiantes universitarios.

\section{Referencias}

Charette, B. (2006). Failing Successfully. Cutter Information. Cutter IT Journal, 17(3).

Chamoun, Y. (2002). Administración profesional de Proyectos: Una guía práctica para programar el éxito en sus proyectos. McGraw Hill.

Dirección General de Innovación, Servicios \& Comercio Interior. (2015). Diagnóstico 2015 S151 Programa para el Desarrollo de la Industria del Software y la Innovación. Recuperado de: http://transparenciapresupuestaria.gob.mx/work/models/PTP/R eingenieria_Gasto/imagenes/Ventanas/Ramo_10/10S151.pdf

Evergreen PM. (2015). Standish Group 2015 Chaos Report. Recuperado de: http://www.evergreenpm.com/standish-group2015-chaos-report/

Gido, J. \& Clements, J. (1999). Administración Exitosa de Proyectos, THOMSON.

Guerra L. \& Bedini Alejandro (2005). Gestión de Proyectos de Software. UTFSM.

Hastie, S. \& Wojewoda, S. (2015). Standish Group 2015 Chaos Report - Q\&A with Jennifer Lynch. EU: Infoq. Recuperado de: http://www.infoq.com/articles/standish-chaos-2015 
Hidding, J. \& Nicholas, J. (2009). Reducing I.T. Project Management Failures: A Research Proposal. Proceedings of the 42nd Hawaii International Conference on System Sciences

Horine, G. (2013). Absolute beginner's guide to project management, Indianapolis, EU: Que.

Humphrey, W. (2005). Why Big Software Projects Fail:The 12 Key Questions. CROSSTALK the Journal of Defense Software Engineering. 18(3), 25-29.

Information and Telecommunication Technologies (ICT) Data and Statistics Division. (2017). Measuring the Information Society Report 2017. - Volume 1. Recuperado de: https://www.itu.int/en/ITU-

D/Statistics/Documents/publications/misr2017/MISR201 7_Volume1.pdf

Lázaro, J. (2015). Adictted to Chaos (2015 Chaos Report by The Standish Group). Recuperado de: http://javierlazarogaspar.blogspot.mx/2015/10/adicttedto-chaos-2015-chaos-report-by.html

Mujica, R. (2014). La Acción Didáctica. Revista TecnológicaEducativa Docentes 2.0. Mayo, 2014. ISSN: 2665-0266. ISBN: 978-980-12-9601-0. Recuperado el 13 de julio de 2020 en: https://ojs.docentes20.com/index.php/revistadocentes20/issue/view/6

Palacios, J., Flores-Roux, E., García, A. (2013). Diagnóstico del sector TIC en México Conectividad e inclusión social para la mejora de la productividad y el crecimiento económico. Banco Interamericano de Desarrollo. Recuperado el 10 de julio de 2020 en: https://imco.org.mx/wpcontent/uploads/2013/1/diagnostic osectorticenmexico_sept2012_2.pdf

Pereira, J., Cerpa, N., \& Rivas, M. (2004). Factores de éxito en proyectos de desarrollo de Software: Análisis de la Industria Chilena del Software. Workshop de Ingeniería de Software, Departamento de Ingeniería de Sistemas. Universidad de Talca.

Project Management Institute. (2013). A guide to the project management body of knowledge (PMBOK guide). Pennsylvania, EU: Project Management Institute, Inc.

Procaccino, J., Verner J. \& Overmyer S. (2002). Case Study: Factors for Early Prediction of Software Success \& Failure. College of Information Science \& Technology, Drexel University. Recuperado el 13 de julio de 2020 en: http://citeseerx.ist.psu.edu/viewdoc/download?doi=10.1.1.475.5 $197 \&$ rep=rep $1 \&$ type $=$ pd

Procaccino, J. \& Verner J. (2007). Software project managers and project success: An exploratory study. The Journal of Systems and Software, 79, 1541-1551.

Rivera, F. \& Hernández G. (2010). Administración de Proyectos, Guía para el aprendizaje. Ed. Pearson.
Rodríguez, E. (2015). Entendiendo el mercado y los negocios TIC. Recuperado el 09 de julio de 2020 en: http://amiti.org.mx/wpcontent/uploads/2015/02/Perspectivas_negocios_mercados_TIC_2015.pdf

Saavedra, M.L. \& Tapia, B. (2013). El uso de las tecnologías de información y comunicación TIC en las micro, pequeñas y medianas empresas (MIPyME) industriales mexicanas. Enl@ce Revista Venezolana de Información, Tecnología y Conocimiento, 10 (1), 85-104.

The Standish Group. (1994). The Chaos Report. Recuperado el 08 de julio de 2020 en: https://www.standishgroup.com/sample_research_files/ch aos_report_1994.pdf

The Standish Group. (2009). Chaos Summary 2009. The 10 laws of Chaos. Recuperado el 08 de julio de 2020 en: https://www.classes.cs.uchicago.edu/archive/2014/fall/51 210-1/required.reading/Standish.Group.Chaos.2009.pdf

The Standish Group. (2013). Chaos Manifesto 2013: Think Big, Act Small. Recuperado el 08 de julio de 2020 en: http://athena.ecs.csus.edu/ buckley/CSc231_files/Standis h_2013_Report.pdf

Weber, E. (2015). Key Lessons from Standish's 2015 Chaos Report. Recuperado el 28 de junio de 2020 en: http://www.erikweberconsulting.com/blog/chaos2015

Zavala J. (2004). ¿Por Qué Fracasan los Proyectos de Software?; Un Enfoque Organizacional. Congreso Nacional de Software Libre 2004. Congreso llevado a cabo en la Universidad Autónoma Metropolitana-Iztapalapa. 